\title{
Prevalência de diabetes melito e tolerância à glicose diminuída na população urbana de 30 a 79 anos da cidade de São Carlos, São Paulo
}

\author{
Prevalence of diabetes and impaired glucose tolerance in the urban \\ population of 30 to 79 years of the city of São Carlos, São Paulo
}

Paula Lima Bosi', Arlety Morais Carvalho², Daniele Contrera², Guilherme Casale ${ }^{2}$, Marina Alexandre Pereira ${ }^{2}$, Matheus Ferreira Gronner², Thatiana Melo Diogo², Maria Tereza da Costa Gonçalves Torquarto, Jorge Oishi ${ }^{3}$, Angela Merice de Oliveira Leal ${ }^{2}$

1 Departamento de Fisioterapia, Universidade Federal de São Carlos, (UFSCar) ${ }^{2}$ Departamento de Medicina, UFSCar ${ }^{3}$ Departamento de Estatística, UFSCar, São Carlos, SP, Brasil
Correspondência para: Angela Merice de Oliveira Leal Departamento de Medicina da UFSCar

Rodovia Washington Luís, km 235 13565-905 - São Carlos, SP, Brasil angelaleal@ufscar.br

Recebido em 25/Jan/2009 Aceito em 29/Mar/2009

\section{RESUMO}

Objetivo: Estimar a prevalência de diabetes melito (DM) e tolerância à glicose diminuída (TGD) na população urbana de 30 a 79 anos da cidade de São Carlos, São Paulo. Métodos: Foi realizado estudo de base populacional entre agosto de 2007 e junho de 2008. Todos os indivíduos, exceto mulheres grávidas, não diabéticos e aqueles com glicemia capilar em jejum $\leq 199 \mathrm{mg} / \mathrm{dl}$ foram submetidos a teste oral de tolerância à glicose e classificados em diabéticos, com TGD ou com tolerância normal à glicose. Resultados: Participaram da pesquisa 1.116 voluntários. As prevalências gerais de DM eTGD foram 13,5\% e 5\%, respectivamente. Houve associação entre DM e TGD e as variáveis "idade", "escolaridade", "índice de massa corpórea" e "circunferência abdominal". Não houve associação entre DM ou TGD e as variáveis "gênero", "cor da pele" e "rendimento mensal". Conclusões: Houve aumento na prevalência de DM em comparação a estudos anteriores no Brasil e na região. Embora tenha havido avanços no diagnóstico, o tratamento do DM requer otimização. Arq Bras Endocrinol Metab. 2009;53(6):726-32

Descritores

Diabetes melito; glicose; prevalência

\section{ABSTRACT}

Objective: To assess the prevalence of diabetes mellitus (DM) and impaired glucose tolerance (IGT) in the urban population aged 30-79 years of the city of São Carlos, São Paulo, Brazil. Methods: It was performed a population survey, from August 2007 to June 2008. Non diabetic individuals, excluding pregnant women, and those with fasting capillary glycemia $\leq 199 \mathrm{mg} / \mathrm{dl}$ were administered oral glucose tolerance test and classified as diabetic, with IGT or with normal glucose tolerance. Results: The number of individuals who participated in the study was 1,116. The overall rates of DM and IGT were $13.5 \%$ and $5 \%$, respectively. DM and IGT were associated with age, education, body mass index and waist. They were not associated with gender, race or income. Conclusions: The prevalence of DM has increased when compared with previous studies in Brazil and in the state. Improvement in the diagnosis has occurred, however, treatment requires optimization. Arq Bras Endocrinol Metab. 2009;53(6):726-32

\section{Keywords}

Diabetes mellitus; glucose; prevalence

\section{INTRODUÇÃO}

$\mathrm{O}$ diabetes melito (DM) tem sido considerado uma das grandes epidemias mundiais do século $21 \mathrm{e}$ um importante problema de Saúde Pública nos países desenvolvidos e, em especial, nos países em desenvolvimento. Em termos mundiais, cerca de 30 milhões de 
indivíduos apresentavam DM em 1985, passando para 135 milhões em 1995 e 240 milhões em 2005, com projeção de atingir 366 milhões em 2030, dos quais dois terços habitarão países em desenvolvimento (1).

Nas Américas, o número de indivíduos com DM foi estimado em 35 milhões para o ano de 2000 e projetado para 64 milhões em 2025 (2). Em estudo realizado na Cidade do México, em 1994, a prevalência de diabetes já alcançava $14,9 \%$ da população adulta (3).

Na população norte-americana com mais de 20 anos, a prevalência de DM, a partir dos dados do National Health and Nutrition Examination Survey (NHANES), de 1999 a 2004, foi estimada em 7,8\%. Essa prevalência foi maior na população feminina e nos indivíduos com idade entre 40 e 59 anos (4).

No Brasil, em estudo multicêntrico avaliando a população urbana de 30 a 69 anos, ao final dos anos 1980, a prevalência de DM era 7,6\%, incidindo igualmente nos dois sexos, mas aumentando com a idade e a adiposidade corporal. As maiores taxas foram observadas em cidades como São Paulo (SP) e Porto Alegre (RS), sugerindo a influência da urbanização e industrialização (5).

Na região nordeste do Estado de São Paulo, na cidade de Ribeirão Preto, a prevalência de DM relatada em 2003 foi de $12 \%$ (6).

De acordo com o Expert Committee on the Diagnosis and Classification of Diabetes Mellitus (7), há um grupo intermediário de indivíduos em que, embora os níveis de glicose não atinjam critérios para o diagnóstico de diabetes, são demasiadamente elevados para serem considerados normais. Nesse grupo, estão os indivíduos com tolerância à glicose diminuída (TGD), que são assim definidos quando, ao teste oral de tolerância à glicose (TOTG), apresentam glicemia final entre 140 e $199 \mathrm{mg} / \mathrm{dl}$. No estudo DECODE (8), foi demonstrado que, além do DM, a TGD também constitui um fator de risco para eventos cardiovasculares. Estima-se que, aproximadamente, 35 a 45 milhões de adultos que apresentam TGD apresentam risco para o desenvolvimento de DM (9).

O DM é uma doença metabólica, cujo marco clínico é a hiperglicemia. As principais formas clínicas de DM são o tipo 1 e o 2. O DM tipo 1 (DMl) decorre da destruição imunológica das células- $\beta$ pancreáticas e é responsável por aproximadamente $10 \%$ dos casos. O DM tipo 2 (DM2) é a forma mais prevalente, correspondendo a mais de $90 \%$ dos casos, e está basicamente associado a dois mecanismos: disfunção de células- $\beta$ e resistência à ação da insulina. Esta última, por sua vez, está intimamente relacionada à obesidade e ao sedentarismo (10).

As consequências do DM, que se instalam a longo prazo, ditas complicações crônicas, decorrem de alterações micro e macrovasculares que levam a disfunção, dano ou falência de vários órgãos e incluem a nefropatia, com elevada taxa de evolução para insuficiência renal, a retinopatia, principal causa de cegueira adquirida, a neuropatia, principal causa não traumática de amputação de membros inferiores, manifestações de disfunção do sistema nervoso autônomo e disfunção sexual (11-14).

Esse quadro incorre em elevado custo econômico e social, comprometendo a produtividade, a qualidade de vida e a sobrevida dos indivíduos diabéticos. Adicionalmente, a associação entre DM e doenças cardiovasculares, que englobam isquemia miocárdica, obstrução arterial periférica e doença vascular cerebral, está bem estabelecida. O DM2 aumenta de duas a quatro vezes o risco de doença cardiovascular, sendo essa a principal causa de mortalidade em diabéticos $(15,16)$.

A identificação dos fatores de risco cardiovascular, em especial, os distúrbios metabólicos, é estratégica na prevenção da morbimortalidade por doenças cardiovasculares, e os estudos epidemiológicos podem propiciar o melhor planejamento de cuidados e recursos destinados à implantação de projetos e medidas que visem à prevenção, ao diagnóstico precoce e ao tratamento eficaz.

O objetivo deste estudo foi estimar a prevalência de DM e TGD na população urbana de 30 a 79 anos da cidade de São Carlos, São Paulo, e suas relações com variáveis sociodemográficas e de saúde.

\section{MÉTODOS}

Neste estudo transversal de base populacional, os dados foram coletados por meio de visitas domiciliares, no período de agosto de 2007 a junho de 2008, na cidade de São Carlos, localizada no centro do Estado de São Paulo, região sudeste do Brasil. A amostra foi constituída por indivíduos de 30 a 79 anos, de ambos os sexos, excluindo mulheres grávidas.

Para a seleção de uma amostra representativa da zona urbana do município, foi utilizado um plano de amostragem probabilística em múltiplos estágios, estratificado segundo as variáveis "renda familiar" e "grau de escolaridade" do chefe da família.

De posse dos dados dos setores, eles foram avaliados em relação à "renda familiar" e ao "grau de escolaridade", formando oito estratos mais homogêneos. De 
cada extrato, foi selecionada uma amostra de setores censitários de tamanho proporcional ao tamanho de cada extrato. Todos os setores censitários foram previamente visitados por uma equipe de entrevistadores para a enumeração das residências, permitindo a atualização dos setores. A amostra final de residências foi selecionada dessa listagem, de forma aleatória.

Para que os elementos de interesse fossem indivíduos na faixa etária de 30 a 79 anos, foram considerados os dados da Fundação Sistema Estadual de Análise de Dados (Seade) para a determinação do tamanho da amostra de residências para atender a essa faixa etária.

O tamanho mínimo da amostra, aproximadamente 1.068 pessoas, foi calculado com auxílio de fórmula estatística, estabelecendo-se $95 \%$ de grau de confiança e margem de erro de $3 \%$. Considerando-se o baixo índice de não participação observado em estudos anteriores, nesta população, o número total da amostra foi estimado em 1.100 pessoas. Como, em média, cada residência, segundo dados do Instituto Brasileiro de Geografia e Estatística (IBGE), possui duas pessoas na faixa etária de interesse, foi feita a seleção de uma amostra de 650 residências, contando com reserva adicional para eventuais substituições. Em caso de haver recusas, foi estabelecido que haveria imediata substituição da casa por seu vizinho do lado direito.

Todos os entrevistadores e examinadores foram alunos de graduação dos cursos da área da Saúde (Medicina, Fisioterapia, Enfermagem e Educação Física), da Universidade Federal de São Carlos (UFSCar). Todos foram previamente treinados e contaram com um coordenador nas atividades de campo.

Este estudo foi aprovado pelo Comitê de Ética em Pesquisa com Seres Humanos da UFSCar.

A população local foi previamente informada sobre esse estudo por meio da imprensa falada e escrita.

Os dados foram coletados em dois dias consecutivos. No primeiro dia, os pesquisadores iam às residências para explicar os objetivos do projeto e convidar os indivíduos a participarem. Caso aceitassem participar, eles respondiam a um questionário, abordando questões sociodemográficas e de Saúde, e eram orientados a permanecer em jejum por 10 a 12 horas para a coleta de amostras de sangue capilar no dia seguinte.

No segundo dia, todos os indivíduos que afirmassem não ser diabéticos e que apresentassem glicemia capilar (GC) em jejum $\leq 199 \mathrm{mg} / \mathrm{dl}$ eram imediatamente submetidos a TOTG, com ingestão de $75 \mathrm{~g}$ de glicose anidra diluída em $300 \mathrm{ml}$ de água. Após 120 minutos, realizava-se outra medida de GC. Os indivíduos que realizavam algum tipo de tratamento para diabetes ou que relatavam já ter apresentado anteriormente glicemia em jejum $\geq 140 \mathrm{mg} / \mathrm{dl}$, ou em qualquer ocasião, acima de $200 \mathrm{mg} / \mathrm{dl}$ não realizavam o TOTG e eram considerados diabéticos. Os indivíduos que, eventualmente, utilizassem medicação oral para prevenção de DM eram instruídos a não fazer uso do medicamento por uma semana para realização do teste.

Os indivíduos que possuíam GC, em jejum ou após TOTG, $\geq 200 \mathrm{mg} / \mathrm{dl}(11,1 \mathrm{mmol} / \mathrm{l})$ foram considerados diabéticos. Indivíduos com GC, após TTOG, entre 140 e $200 \mathrm{mg} / \mathrm{dl}(7,7$ e $11,1 \mathrm{mmol} / \mathrm{l})$ foram considerados como portadores de TGD e aqueles com GC, após TOTG, até $140 \mathrm{mg} / \mathrm{dl}(7,7 \mathrm{mmol} / \mathrm{l})$ foram considerados como tendo tolerância normal à glicose (17). A glicemia foi determinada por medidores portáteis $\left(\right.$ CardioChek $\left.^{\circledR}\right)$ para leitura imediata.

A altura e a circunferência abdominal (CA) foram medidas com fita métrica e o peso, aferido com balança digital portátil. A CA foi medida no ponto médio entre o último arco costal e a crista ilíaca.

O teste do $\chi^{2}$ foi utilizado para análise dos coeficientes de prevalência. $\mathrm{O}$ nível de significância adotado foi $\mathrm{p}<0,05$.

\section{RESULTADOS}

No total, 1.116 voluntários participaram da pesquisa, sendo 720 (65\%) mulheres e 396 (35\%) homens. A média de idade foi $52,54 \pm 13,74$ anos. A taxa de recusa foi 4,5\%.

De acordo com os critérios utilizados, 862 indivíduos foram classificados como possuindo tolerância normal à glicose; 69, como possuindo TGD; e 185, como portadores de DM, o que corresponderia a prevalências de DM e TGD iguais a 16,6\% e 6,2\% da população, respectivamente. Após ajuste por idade das taxas de prevalência a partir dos dados populacionais de São Carlos (IBGE, 2008) (18) por método direto de padronização, as prevalências gerais de DM e TGD foram $13,5 \%$ e $5 \%$, respectivamente (Tabela 1 ).

Em relação à idade, a prevalência de DM e TGD foi $4,7 \%$ e $1,2 \%$ na faixa etária de 30 a 39 anos, e $29 \%$ e $13,2 \%$ na faixa etária de 70 a 79 anos, respectivamente, havendo associação entre as variáveis "faixa etária" e "DM" e "TGD" ( $<<0,05)$ e aumento crescente da prevalência de DM e TGD com o aumento da idade. Não foi observada associação quanto à distribuição por gênero (Tabelas 1 e 2). 


\begin{tabular}{|c|c|c|c|c|}
\hline \multirow{2}{*}{$\begin{array}{l}\text { Faixa etária } \\
\text { (anos) }\end{array}$} & \multicolumn{2}{|c|}{ DM (IC) } & \multicolumn{2}{|c|}{ TGD (IC) } \\
\hline & $\%$ & $\mathbf{n}$ & $\%$ & $\mathbf{n}$ \\
\hline 30 a 39 & 4,7 & 11 & 1,2 & 3 \\
\hline 40 a 49 & 10 & 25 & 5,2 & 13 \\
\hline 50 a 59 & 18,8 & 53 & 5,3 & 15 \\
\hline 60 a 69 & 26 & 48 & 8,6 & 16 \\
\hline 70 a 79 & 29 & 48 & 13,2 & 22 \\
\hline Total & 16,6 & 185 & 6,2 & 69 \\
\hline Ajuste IBGE/2008 & $13,5(8,5-18,5)$ & & $5(0-10,2)$ & \\
\hline
\end{tabular}

IC: intervalo de confiança.

${ }^{*} p<0,05$.

Tabela 2. Prevalência de DM de acordo com a faixa etária e 0 gênero

\begin{tabular}{|c|c|c|c|c|}
\hline \multirow{2}{*}{ Faixa etária } & \multicolumn{2}{|c|}{ Feminino (IC) } & \multicolumn{2}{|c|}{ Masculino (IC) } \\
\hline & $\%$ & $\mathbf{n}$ & $\%$ & $\mathbf{n}$ \\
\hline 30 a 39 & 4,5 & 7 & 5 & 4 \\
\hline 40 a 49 & 11 & 18 & 8 & 7 \\
\hline 50 a 59 & 21 & 38 & 15 & 15 \\
\hline 60 a 69 & 27 & 31 & 25 & 17 \\
\hline 70 a 79 & 34 & 35 & 21 & 13 \\
\hline Total & 17,9 & 129 & 14,1 & 56 \\
\hline Ajuste IBGE/2008 & $15(9,8-20,2)$ & & $11,2(6,6-15,8)$ & \\
\hline
\end{tabular}

IC: intervalo de confiança.

Na tabela 3, as prevalências de DM e TGD são apresentadas de acordo com a cor da pele (autorreferida), o rendimento mensal, o grau de escolaridade, o índice de massa corpórea (IMC, $\mathrm{kg} / \mathrm{m}^{2}$ ) e a CA ( $\left.\mathrm{cm}\right)$. Não houve associação entre as taxas de DM e TGD e cor da pele ou rendimento mensal. Entretanto, os indivíduos com escolaridade até o ensino fundamental apresentaram prevalência de DM maior do que aqueles com escolaridade igual ou superior ao ensino médio/superior.

Os indivíduos com aumento do peso (sobrepeso e obesidade, IMC $\geq 25 \mathrm{~kg} / \mathrm{m}^{2}$ ) apresentaram prevalência de DM maior do que os indivíduos com peso normal. Houve associação significativa entre as prevalências de DM e TGD e CA, tanto no sexo masculino quanto no sexo feminino. A prevalência de DM é maior (12,9\%) nos indivíduos que apresentam aumento da CA do que naqueles com CA normal $(3,7 \%)$, considerando como limites normais $102 \mathrm{~cm}$ no sexo masculino e $88 \mathrm{~cm}$ no sexo feminino (19).

Dos 185 indivíduos portadores de DM, 157 (85\%) já haviam sido previamente diagnosticados e 28 (15\%) foram detectados neste estudo. Neste último grupo, 7 (25\%) apresentaram GC em jejum $\geq 200 \mathrm{mg} / \mathrm{dl}$ e 21 (75\%) foram classificados após TOTG.
Tabela 3. Prevalência de DM e TGD (número de indivíduos e \%) de acordo com o rendimento mensal, cor da pele, grau de escolaridade, IMC e CA.

\begin{tabular}{|c|c|c|c|c|c|}
\hline & & \multicolumn{2}{|c|}{ DM } & \multicolumn{2}{|c|}{ TGD } \\
\hline & & $\mathbf{n}$ & (\%) & $\mathbf{n}$ & (\%) \\
\hline \multirow{6}{*}{$\begin{array}{l}\text { Rendimento } \\
\text { mensal } \\
\text { (salários } \\
\text { mínimos) }\end{array}$} & Até 2 & 31 & 14,6 & 12 & 6 \\
\hline & 2 a 5 & 114 & 18,2 & 31 & 5 \\
\hline & 5 a 10 & 31 & 15,6 & 15 & 7 \\
\hline & 10 a 20 & 7 & 14 & 6 & 10 \\
\hline & Acima de 20 & 2 & 11,1 & 2 & 11 \\
\hline & Total & 185 & 13,5 & 69 & 5 \\
\hline \multirow{3}{*}{ Cor da pele } & Branca & 125 & 16,7 & 53 & 5,4 \\
\hline & Não branca & 60 & 16 & 16 & 7 \\
\hline & Total & 185 & 13,5 & 69 & 5 \\
\hline \multirow{3}{*}{$\begin{array}{l}\text { Grau de } \\
\text { escolaridade* }\end{array}$} & Fundamental & 139 & 19 & 42 & 5,4 \\
\hline & Médio/superior & 46 & 9,8 & 27 & 7 \\
\hline & Total & 185 & 13,5 & 69 & 5 \\
\hline \multirow{3}{*}{$\mathrm{IMC}^{*}$} & Normal $\left(<24,9 \mathrm{~kg} / \mathrm{m}^{2}\right)$ & 20 & 4,8 & 20 & 5,1 \\
\hline & $\begin{array}{l}\text { Sobrepeso/obesidade } \\
\left(\geq 25 \mathrm{~kg} / \mathrm{m}^{2}\right)\end{array}$ & 165 & 22,5 & 49 & 6,8 \\
\hline & Total & 185 & 13,5 & 69 & 5 \\
\hline \multirow{3}{*}{$C A^{*}$} & $\begin{array}{l}\text { Normal }(<102 \mathrm{~cm} \\
\text { em homens e }<88 \mathrm{~cm} \\
\text { em mulheres) }\end{array}$ & 41 & 3,7 & 24 & 2,15 \\
\hline & Aumentada & 144 & 12,9 & 45 & 4 \\
\hline & Total & 185 & 13,5 & 69 & 5 \\
\hline
\end{tabular}

*diferença entre os constituintes da variável, $\mathrm{P}<0,05$.

Dos 157 indivíduos previamente diagnosticados como diabéticos, $142(90,5 \%)$ estavam em tratamento. Destes, $13(9,1 \%)$ estavam sendo tratados somente com dieta, $46(32,3 \%)$ utilizavam antidiabético oral (ADO), $4(2,8 \%)$ utilizavam insulina, $42(29,5 \%)$ utilizavam $\mathrm{ADO}$ associado à dieta, $18(12,6 \%)$ utilizavam $\mathrm{ADO}$ associado à insulina e $19(13,7 \%)$ utilizavam dieta, $\mathrm{ADO}$ e insulina (Figura 1 ).

De acordo com a GC em jejum, os indivíduos em tratamento foram classificados em controlados $(\mathrm{GC}<$ $120 \mathrm{mg} / \mathrm{dl}$ ou $6,7 \mathrm{mmol} / \mathrm{l})$ e não controlados (GC $\geq$ $120 \mathrm{mg} / \mathrm{dl}$ ou $\geq 6,7 \mathrm{mmol} / \mathrm{l})$. Assim, $66(46 \%)$ foram considerados controlados e 76 (54\%), não controlados.

\section{DISCUSSÃO}

Neste estudo, as prevalências de DM e TGD corresponderam, respectivamente, a $13,5 \%$ e $5 \%$ da população urbana de São Carlos com idade entre 30 e 79 anos.

Em estudo recente, Ong e cols. (4) analisaram a tendência de evolução da prevalência e controle de 


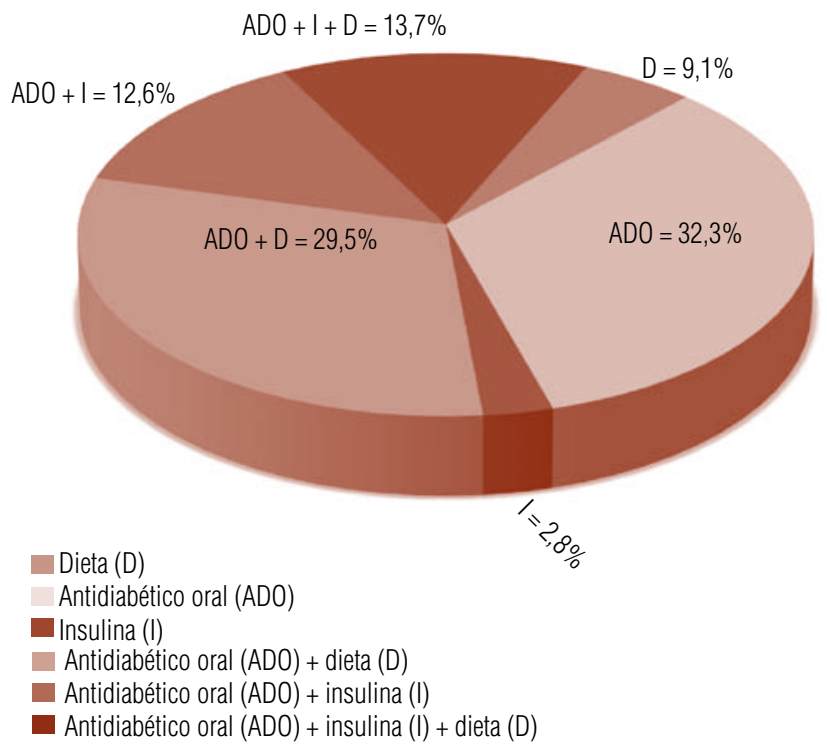

Figura 1. Distribuição percentual do tipo de tratamento utilizado pelos indivíduos diabéticos $(n=142)$.

DM por meio dos dados do NHANES, no período compreendido entre 1999 e 2004. Os autores observaram que a prevalência geral de DM na população dos Estados Unidos não aumentou significativamente entre os anos 1999 e $2000(6,4 \%)$ e 2003 e $2004(7,8 \%)$. Entretanto, houve aumento significativo da prevalência de DM entre indivíduos com idade entre 40 e 59 anos, mulheres, brancos não hispânicos e obesos.

No Brasil, os estudos de base populacional são escassos, o que dificulta análises comparativas. Entre os anos de 1986 e 1988, foi realizado estudo multicêntrico que estimou a prevalência de DM e TGD na população brasileira em 7,6\% e 7,8\%, respectivamente. Neste estudo multicêntrico, foram observadas diferenças regionais de prevalência, tendo sido observada a maior taxa de prevalência de DM em São Paulo (9,7\%), o maior pólo industrial do país (5). Desde então, não se dispõe de dados de estudos de abrangência nacional para comparação.

No estudo realizado em Ribeirão Preto, no interior do Estado de São Paulo, distante aproximadamente 100 km de São Carlos, na década de 1990, as taxas de prevalência de DM e TGD (12,1\% e 7,7\%, respectivamente) atingiram valores superiores àqueles observados no estudo multicêntrico realizado uma década antes (6). Embora haja diferenças metodológicas entre os estudos, os resultados apresentados aqui confirmam, como esperado previamente, aumento da prevalência de DM no país e, em particular, no Estado. A atual taxa de prevalência de DM observada em São Carlos é compatível com importante aumento da industrialização e urbanização na cidade (5).
Não foi encontrada associação entre DM e cor da pele dos indivíduos estudados. O mesmo foi observado em outros estudos brasileiros $(5,6,20)$, nos quais a prevalência da doença em brancos e não brancos foi semelhante. Estudos recentes têm demonstrado prevalência de DM maior em latino-americanos do que em caucasianos, todos residentes nos Estados Unidos. Nessa população, os índices de DM são quase o dobro do que em outras populações. Embora diferenças étnicas e fatores genéticos possam estar envolvidos, os fatores ambientais parecem ser preponderantes (2l). A população brasileira tem como característica a miscigenação, o que torna difícil a comparação com outras populações.

A prevalência de DM e TGD não foi diferente entre homens ( 14,1 e $7 \%$, respectivamente) e mulheres (17,9 e $5,6 \%$, respectivamente), resultado semelhante ao descrito em outros estudos $(5,6)$.

A prevalência de DM aumentou com o aumento da idade, descrevendo uma curva ascendente, com $6 \%$ de diabéticos dos 30 aos 39 anos e $26 \%$ dos 70 aos 79 anos. Este crescimento da prevalência do DM com a idade se mantém, como observado em estudos prévios $(5,6)$.

Neste estudo, foi encontrada maior prevalência de DM em indivíduos com menor grau de escolaridade. A associação entre escolaridade e prevalência de DM também foi observada antes, tanto no Brasil como nos Estados Unidos, na Europa e na Ásia (4,22-25). O grau de escolaridade é um dos indicadores socioeconômicos mais importantes, capaz de influenciar o autocuidado e o acesso às medidas preventivas e terapêuticas em Saúde (25).

Observou-se associação entre a prevalência de DM e IMC e CA, o que também foi encontrado em estudos anteriores (6). O papel da obesidade, em especial, a obesidade visceral, como fator predisponente para o desenvolvimento de DM e demais fatores de risco cardiovascular, está bem estabelecido (26). A ligação entre obesidade e diabetes é mediada pelos efeitos da resistência à insulina. Para que a obesidade e a resistência à insulina culminem em DM2, entretanto, as célulaspancreáticas devem ser incapazes de compensar a sensibilidade diminuída à insulina $(27,28)$.

Neste estudo, $85 \%$ dos indivíduos diabéticos já possuíam diagnóstico prévio, enquanto no estudo realizado em Ribeirão Preto (6), há aproximadamente uma década, essa taxa era de $75 \%$. Isto pode indicar maior eficiência dos sistemas de Saúde em diagnosticar DM e das medidas de educação em Saúde com a população e os profissionais de Saúde. 
Com relação ao tipo de tratamento utilizado pelos indivíduos diabéticos dessa amostra, estes utilizavam, em sua maioria, $\mathrm{ADO}$, isoladamente ou associado à dieta. Embora mais de $90 \%$ dos diabéticos fizessem algum tipo de tratamento, a maioria não se encontrava metabolicamente compensada. Elevada taxa de mau controle metabólico entre pacientes diabéticos, em tratamento, também foi encontrada por Assunção e cols. (29). Esse resultado indica a necessidade de esforços para otimizar o controle glicêmico e prevenir, assim, as complicações crônicas $(10,30,31)$.

\section{CONCLUSÕES}

As prevalências de DM e TGD na população urbana de São Carlos com idade de 30 a 79 anos foram, respectivamente, $13,5 \%$ e $5 \%$. A prevalência de DM é, portanto, superior àquela observada em estudos nacional e regional prévios. Envelhecimento, obesidade e baixa escolaridade são fatores associados à elevada prevalência de DM. Embora tenha havido avanços no diagnóstico, o tratamento do DM requer otimização.

Agradecimentos: à Fundação de Amparo à Pesquisa do Estado de São Paulo (Fapesp) e à Pró-Reitoria de Extensão da UFSCar.

Declaração: os autores declaram não haver conflitos de interesse científico neste estudo.

\section{REFERÊNCIAS}

1. Wild S, Roglic G, Green A, Sicree R, King H. Global prevalence of diabetes. Estimates for the year 2000 and projections for 2030. Diabetes Care. 2004;27(5):1047-53.

2. King H, Aubert RE, Herman WH. Global burden of diabetes, 19952025: prevalence, numerical estimates, and projections. Diabetes Care. 1998;21:1414-31.

3. Barceló A, Rajpathak S. Incidence and prevalence of diabetes mellitus in the Americas. Rev Panam Salud Publica. 2001;10(5):300-8.

4. Ong KL, Cheung BM, Wong LY, Wat NM, Tan KC, Lam KS. Prevalence, treatment, and control of diagnosed diabetes in the U.S. National Health and Nutrition Examination Survey 1999-2004. Ann Epidemiol. 2008;18(3):222-9.

5. Malerbi DA, Franco LJ. Multicenter study of the prevalence of diabetes mellitus and impaired glucose tolerance in the urban Brazilian population aged 30-69 yr. The Brazilian Cooperative Group on the Study of Diabetes Prevalence. Diabetes Care. 1992;15(11):1509-16.

6. Torquato MT, Montenegro Jr RM, Viana LA, de Souza RA, Lanna CM, Lucas JC, et al. Prevalence of diabetes mellitus and impaired glucose tolerance in the urban population aged 30-69 years in Ribeirão Preto (São Paulo), Brazil. Sao Paulo Med J. 2003;121(6):224-30.

7. Genuth S, Alberti KG, Bennett P, Buse J, Defronzo R, Kahn R, Kitzmiller J, Knowler WC, Lebovitz H, Lernmark A, Nathan D, Palmer J, Rizza R, Saudek C, Shaw J, Steffes M, Stern M, Tuomilehto J, Zimmet $P$; Expert committee on the diagnosis and classification of diabetes mellitus. Follow-up report on the diagnosis of diabetes mellitus. Diabetes Care. 2003;26(11):3160-7.

8. The DECODE Study Group. European Diabetes Epidemiology Group. Diabetes Epidemiology: Collaborative Analysis of Diagnostic criteria in Europe. Glucose tolerance and mortality: comparison of WHO and American Diabetes Association diagnostic criteria. Lancet. 1999;354(9179):617-21.

9. Roman $\mathrm{SH}$, Harris MI. Management of diabetes mellitus from a public health perspective. Endocrinol Metab Clin North Am. 1997;26(3):443-74.

10. Stumvoll M, Goldstein BJ, Haeften TW.Type 2 diabetes: principles of pathogenesis and therapy. Lancet. 2005;365(9467):1333-46.

11. Molitch ME, DeFronzo RA, Franz MJ, Keane WF, Mogensen CE, Parving $\mathrm{HH}$, Steffes MW; American Diabetes Association. Nephropathy in diabetes (Position Statement). Diabetes Care. 2004;27(Suppl. 1):S79-S83.

12. Fong DS, Aiello L, GardnerTW, King GL, Blankenship G, Cavallerano JD, Ferris FL 3rd, Klein R; American Diabetes Association. Retinopathy in diabetes (Position Statement). Diabetes Care. 2004;27(Suppl. 1):S84-S87.

13. Tesfaye S, Stevens LK, Stephenson JM, Fuller JH, Plater M, lonescu-Tirgoviste $C$, et al. Prevalence of diabetic peripheral neuropathy and its relation to glycaemic control and potential risk factors: the EURODIAB IDDM Complications Study. Diabetologia. 1996;39(11):1677-384.

14. Boulton AJM, Vileikyte L, Ragnarson-Tennvall G, Apelqvist J.The global burden of diabetic foot disease. Lancet. 2005;366(9498):1719-24.

15. Haffner SM. Coronary heart disease in patients with diabetes. $\mathrm{N}$ Engl J Med. 2000;342:1040-2.

16. Grundy SM, Benjamin IJ, Burke GL, Chait A, Eckel RH, Howard $\mathrm{BV}$, et al. Diabetes and cardiovascular disease. A statement for healthcare propessionals from the American Heart Association. Circulation. 1999;100(10):1134-46.

17. [No authors listed]. Report of the Expert Committee on the Diagnosis and Classification of Diabetes Mellitus. Diabetes Care. 1997;20(7):1183-97.

18. IBGE. Estimativas das populações residentes, 2008, Instituto Brasileiro de Geografia e Estatística, http://www.ibge.gov.br/home/ estatistica/populacao/estimativa2008. Acesso em: 2008.

19. Executive Summary of The Third Report of the National Cholesterol Education Program (NCEP). Expert Panel on Detection, Evaluation, and Treatment of High Blood Cholesterol in Adults (Adult Treatment Panel III). JAMA. 2001;285:2486-97.

20. Oliveira JEP, Milech A, Franco LJ. The prevalence of diabetes in Rio de Janeiro, Brazil. The Cooperative Group for the Study of Diabetes Prevalence in Rio de Janeiro. Diabetes Care. 1996;19(6):663-6.

21. Umpierrez GE, Gonzalez A, Umpierrez D, Pimentel D. Diabetes mellitus in the Hispanic/Latino population: an increasing health care challenge in the United States. Am J Med Sci. 2007;334(4):274-82.

22. Maty SC, Everson-Rose SA, Haan MN, Raghunathan TE, Kaplan GA. Education, income, occupation, and the 34-year incidence (1965-99) of type 2 diabetes in the Alameda County Study. Int J Epidemiol. 2005;34(6):1274-81.

23. Espelt A, Borrel C, Roskam AJ, Rodríguez-Sanz M, Stirbu I, Dalmau-Bueno $A$, et al. Socioeconomic inequalities in diabetes mellitus across Europe at the beginning of the 21st century. Diabetologia. 2008;51(11):1971-9.

24. Azimi-Nezhad M, Ghayour-mobarhan M, Parizadeh MR, Safarian M, Esmaeili H, Parizadeh SM, et al. Prevalence of type 2 diabetes mellitus in Iran and its relationship with gender, urbanization, education, marital status and occupation. Singapore Med J. 2008;49(7):571-6. 
25. Steinvil A, Shirom A, Melamed S, Toker S, Justo D, Saar N, et al. Relation of educational level to inflamation-sensitive biomarker level. Am J Cardiol. 2008;102(8):1034-9.

26. VisscherTL, Seidell JC. The public health impact of obesity. Annu Rev Public Health. 2001;22:355-75.

27. Kahn SE, Hull RL, Utzschneider KM. Mechanisms linking obesity to insulin resistance and type 2 diabetes. Nature. 2006;444(7121):840-6.

28. Guilherme A, Virbasius JV, Puri V, Czech MP. Adipocyte dysfunctions linking obesity to insulin resistance and type 2 diabetes. Mol Cell Biol. 2008;9(5):367-77.
29. Assunção MCF, Santos IS, Valle NCJ. Controle glicêmico em pacientes diabéticos atendidos em centros de atenção primária à saúde. Rev Saúde Pública. 2005;39(2):183-90.

30. [No authors listed]. Effect of intensive diabetes management on macrovascular events and risk factors in the Diabetes Control and Complications Trial. Am J Cardiol. 1995;75(14):894-903.

31. UK Prospective Diabetes Study (UKPDS) Group. Intensive bloodglucose control with sulphonylureas or insulin compared with conventional treatment and risk of complications in patients with type 2 diabetes (UKPDS 33). Lancet. 1998;352(9131):837-53. 